\title{
Erratum: Thermodynamics of solid and liquid EAM metals: A variational study [J. Chem. Phys. 94, 5090 (1991)]
}

\author{
R. LeSar \\ Theoretical Division, Los Alamos National Laboratory, Los Alamos, New Mexico 87545 \\ R. Najafabadi and D. J. Srolovitz \\ Department of Materials Science and Engineering, University of Michigan, Ann Arbor, Michigan 48109
}

Due to an error in our computer program, the term in the Ross variational method that approximates a $1 / r^{12}$ reference system with a hard-sphere reference system was inadvertently set to zero (i.e., $F_{12}=0$ in Eq. 12). Thus, all of our results for the liquid metals were calculated with a hard-sphere reference system, not with the Ross procedure. Based on using the hard-sphere reference system, however, the conclusions are unchanged.

Including the Ross correction term improves the agreement between the variational results for the liquid metals and the accurate Monte Carlo results. In our earlier paper, using the first-order theory, we found an average error of $0.97 \%$ in the free energies of the liquid metals $(\mathrm{Ag}, \mathrm{Au}, \mathrm{Cu}, \mathrm{Ni}, \mathrm{Pd}$, and $\mathrm{Pt}$ ) at the melting points determined by Monte Carlo simulations with the same potentials. Including the Ross correction term, we now find an average error of only $0.20 \%$. Including the (approximate) second-order correction gave an average error of $1.52 \%$ before and $0.55 \%$ with the Ross correction term included properly. The accuracy of the calculated internal energies $(E)$ are also greatly improved by using the Ross correction. With the hard-sphere reference system, we found an average error in $E$ at the melting points for the liquid metals of about $0.045 \mathrm{eV} /$ atom or $1.28 \%$. With the Ross procedure, that error is reduced to $0.014 \mathrm{eV} /$ atom or
$0.39 \%$. The entropies calculated from the variational results are, however, in slightly worse agreement with the Ross term included, with the previous error being about $0.47 \%$ and the error with the Ross term about $1.37 \%$.

As noted in our paper, accurate melting points require a high degree of consistency between the solid and liquid results. Our melting points were reasonably accurate when based on the hard-sphere reference state for the liquids largely because of cancellation of errors in the free energies of the solids and liquids. With the more accurate results for the liquid free energies using the Ross procedure, the agreement in the melting points between the variational calculations and the Monte Carlo results are considerably worse. With the hard-sphere reference system, the average error in melting point was about $100 \mathrm{~K}$ or $7.5 \%$, with the variational results equally split above and below the Monte Carlo results. With the more accurate Ross procedure for the liquid metals, that error is about $300 \mathrm{~K}$ or $25 \%$, with all melting points underestimated. Including the secondorder term increased the errors for the hard-sphere calculations to $10 \%$, but lowered the errors in the present results to about $14 \%$. The most reliable melting points thus came from using the first-order hard-sphere results for the liquid-state free energies. 